\title{
On Student Collaboration and Competition in an Inquiry-based Multi-user Communications and Jamming Exercise
}

\section{Kirsten R. Basinet, Western Washington University}

Kirsten R. Basinet is pursuing a master's degree in computer science at Western Washington University, where she graduated in 2016 with a B.S. in electrical engineering. She is passionate about communications systems, broadcast technologies, STEM education, accessibility, and sustainable engineering.

\section{Prof. Andrew G. Klein, Western Washington University}

Andrew G. Klein joined Western Washington University (WWU) in 2014 and is currently an associate professor with a joint appointment to the department of Engineering and Design (Electrical Engineering Program) and the graduate faculty of Computer Science. He received the B.S. degree in electrical engineering from Cornell University, and the M.S. degree in electrical engineering and computer sciences from the University of California, Berkeley. He then worked for awhile at several Silicon Valley startup companies before returning to Cornell to pursue a Ph.D. in electrical and computer engineering in 2006. Prior to his arrival at WWU, he worked as a postdoctoral researcher at Supélec/LSS near Paris, France, and was an assistant professor at Worcester Polytechnic Institute.

\section{Dr. Richard Martin, The Air Force Institute of Technology}

Richard K. Martin received dual B.S. degrees (summa cum laude) in physics and electrical engineering from the University of Maryland, College Park, in 1999 and the M.S. and Ph.D. degrees in electrical engineering from Cornell University, Ithaca, NY, in 2001 and 2004, respectively. Since August 2004, he has been with the Department of Electrical and Computer Engineering, Air Force Institute of Technology (AFIT), Dayton, OH, where he is a Professor. He is the author of 35 journal papers and 68 conference papers, and he holds five patents. His research interests include radio tomographic imaging; navigation and source localization; electronic warfare; and laser radar. Dr. Martin has won seven teaching awards including the Air Force wide award for Outstanding Science and Engineering Educator. He has served as a Senior Area Editor and an Associate Editor for IEEE Signal Processing Letters and as a Guest Editor for The IEEE Journal of Selected Topics in Signal Processing. 


\title{
On Student Collaboration and Competition in an Inquiry-Based Multiuser Communications and Jamming Exercise
}

\begin{abstract}
This paper describes an inquiry-based laboratory exercise used to introduce senior-level electrical engineering students to the concepts of multiuser communication systems. The exercise includes both collaborative and competitive gaming elements, and requires students to share a single transmitter to send messages to multiple users. In the collaborative mode, the students are tasked with maximizing the minimum rate per user over the whole group, while in the competitive mode they are tasked with maximizing their individual rate while possibly jamming their peers. The paper presents results of a survey assessing student opinions of the relative benefits of collaboration and competition in this exercise. While the results depend heavily on student self-reporting, the data suggest that there is value in both the competitive and collaborative elements of this exercise, with some caveats.
\end{abstract}

\section{Introduction}

Active learning has been shown to improve student engagement, academic achievement, and fulfillment of learning outcomes in STEM fields. ${ }^{1,2,3}$. Under the umbrella of active learning, however, a large variety of different (and sometimes contradictory) methodologies have been proposed; including project-based learning, problem-based learning, gamification, tinkering, collaborative learning, class competitions, and many others. As educators become more interested in student-centered pedagogies, the question of which specific techniques are most effective is increasingly important.

Because active learning is still an emerging paradigm, the number of studies examining distinct approaches is somewhat limited, and the difficulty of isolating those techniques in the classroom environment is a recognized concern. ${ }^{1}$ In this paper, we wish to contribute to the growing pool of literature by exploring the duality of collaboration and competition in particular. While there are many allusions to learning via collaboration and competition in the literature - with a general sense that both may be desirable in different contexts - there are very few studies which attempt to compare them directly, especially in the field of electrical engineering education.

This work funded in part by The Office of Naval Research, Award Number N000141512442-P0001. The views expressed in this paper are those of the authors, and do not reflect the official policy or position of the United States Air Force, Department of Defense, or the U.S. Government. This document has been approved for public release; distribution unlimited. 
For years, student collaboration has been recognized as a critical component of active learning, and there is compelling evidence that encouraging students to work together really does have a positive impact on performance, grades, and knowledge retention. ${ }^{1,4,5}$ However, the assumption that collaboration is a defining characteristic of active learning is increasingly questionable. Recent work on gamification in particular has supported the idea that competition can be a powerful student-centered teaching tool in its own right, especially when used to motivate learners, although some results suggest that emphasizing competition too heavily may have a negative effect on participation and learning. ${ }^{3,6,7,8}$

A few studies have attempted to directly examine collaboration and competition in contrast with one another. A literature survey which reviewed work published between 1929-1993 found that cooperative teams outperformed individuals competing with each other on problem-solving tasks. ${ }^{9}$ However, a 1996 study presented qualitative evidence that collaboration and competition were successfully used together in an engineering communications course to improve learning. ${ }^{10}$ A 2014 case study involving two software organizations echoed this finding in an industrial setting, where competition between teams helped to solidify working relationships between team members. ${ }^{11}$ Finally, a 2014 study of massively-multiplayer online games suggested that competition between player factions increased the incentive for collaboration within those groups. $^{12}$

In this work-in-progress paper, we further investigate the impact of inter-group collaboration and competition. We present a novel, inquiry-based lab exercise for introducing students to the principles of multiuser communication systems. The activity makes use of group work, and contains distinct collaborative and competitive gaming elements which we study to better understand which group dynamics motivate students and foster learning.

In the lab exercise, students enrolled in a senior-level digital communications course are tasked with creating a wireless, acoustic digital transceiver using MATLAB and off-the-shelf components. Multiple student groups are given a single shared speaker which serves as a transmitter, broadcasting the summation of all groups' signals. In addition, each group is provided a microphone, which acts as a receiver and allows each group to decode the unique information intended for them. The lab is given during the first week of the course, well before the theoretical concepts of multiuser communications have been covered in lecture, and prompts students to derive their own mechanism for sharing a single transmitter among several users. A visual representation of the multiuser system model is shown in Fig. 1.

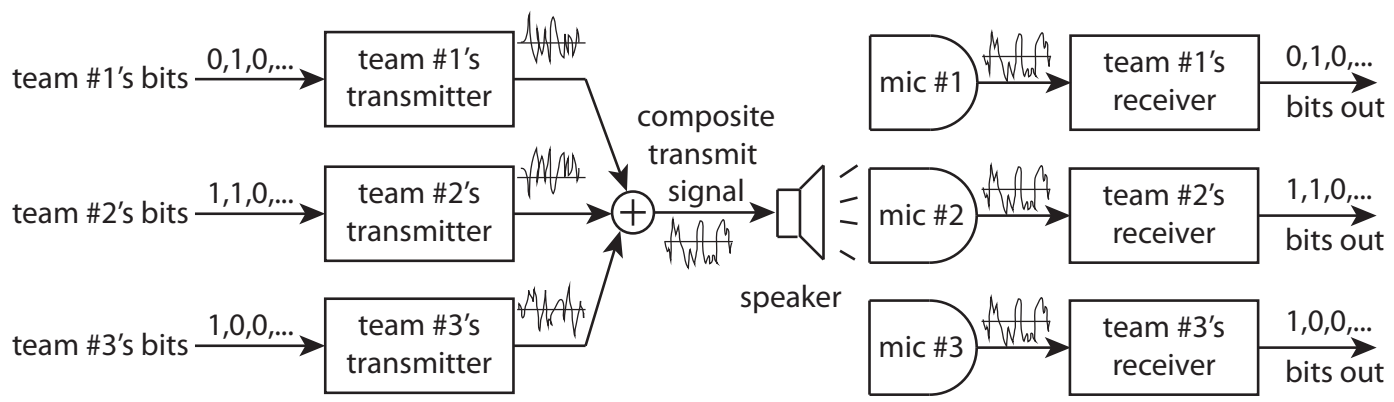

Figure 1: Block diagram of multiuser communication scheme 
The first part of the lab, which is strictly collaborative, is intended to model the downlink in a cellular basestation, wherein teams of student teams are encouraged to work together to make the system as efficient as possible with a design goal of maximizing the minimum rate over all receivers. Collaboration is incentivized by a grading scheme that rewards the entire group an increasing amount of points as the error-free minimum rate increases. The second, competitive part of the lab pits teams against each other, with points assigned based on which team achieves the highest individual data rate, subject to a peak power constraint. The speaker and microphone implicitly impose a bandwidth constraint, as well. Students are encouraged to create robust receivers through software improvements, and are told that they may interfere with their classmates' transmissions by sending a malicious signal. In both parts of the lab, students are not provided with an explicit list of procedures to follow, but are instead prompted to experiment and investigate the best solution on their own.

Indeed, qualitative results from a similar inquiry-based communications lab suggests that the technique described in this paper will be effective when applied to students learning about multiuser communications systems ${ }^{13}$. In the sections that follow, we describe the exercise in detail and discuss both qualitative and quantitative results obtained from a survey presented to students at the end of the course.

\section{Materials}

A wireless acoustic transceiver design was selected since it can be implemented very economically with PCs, a speaker, and microphones. Fortunately, while the acoustic transmission medium and hardware have little similarity with RF transmission, the mathematical descriptions of signals and concepts such as modulation in acoustic communication are identical to those in conventional electromagnetic RF wireless communication. Thus, by conducting experiments using acoustic hardware in the lab, the students are able to hear the signals they create, which provides a less abstract observation of the operation of wireless communication systems. In addition, since acoustic frequencies are much lower, the labs can be completed with much slower sampling rates, and with readily available hardware without sacrificing learning. Furthermore, the use of audio signals removes concern for regulatory (e.g. FCC) issues that arise when transmitting RF signals.

The materials used for this lab consisted of very inexpensive off-the-shelf components. The single transmitter was implemented using a PC with MATLAB and a set of Logitech speakers (\$20.00). Similarly, the receivers provided to each pair of students included a PC with MATLAB and a General Electric 98950 detachable desktop microphone (\$11.00 ea). We note that Octave would work just as well in place of MATLAB.

The setup of the speaker and microphone is shown in Fig. 2. Since no constraints were placed on transmission distance, students generally placed their microphones 1-2' from the microphone to maximize signal-to-noise ratio (SNR). 


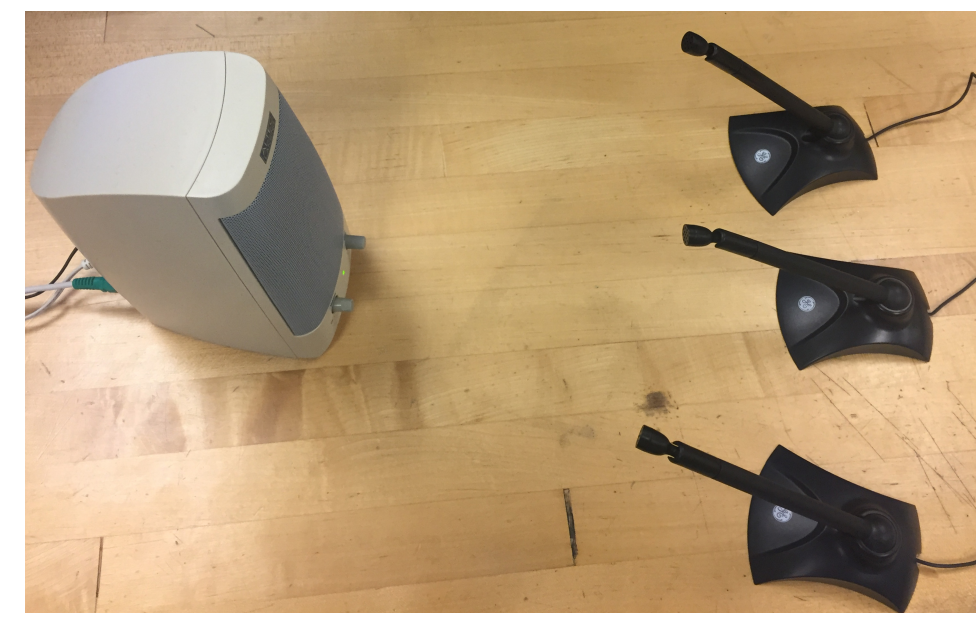

Figure 2: Experimental Setup of Multiuser Acoustic Transmitter and Three Receivers

\section{Implementation}

Western Washington University (WWU) has recently started a degree program in electrical engineering (EE), and in the conception of this new program, hands-on and project-based learning were identified as key components of the curriculum. As such, the senior-level digital communications course (EE 460) has a weekly lab, as do all but 2 of the 30 courses in the program. This exercise was given to the students as the first lab of the quarter, before the students had been exposed to multiuser system concepts. Students entering this digital communications course at WWU had all taken at least two quarters of signals and systems, one previous communications systems course, and all had significant exposure to MATLAB, time and frequency domain representations of signals, linear time-invariant systems, and Fourier theory. In addition, the students had all completed a single-user, point-to-point laboratory exercise in a previous quarter that required them to implement transmitters and receivers in MATLAB using similar acoustic hardware ${ }^{13}$.

By drawing upon their prior knowledge of single-user point-to-point communications, this lab prompted them to discover a way to share a single transmitter to wirelessly send $N$ unique messages to $N$ different receivers. The students were told that there was no one right answer to the lab, that they should not hesitate to ask questions of the instructor, and that moments of frustration are natural in open-ended discovery. The students had two 110-minute lab periods to complete their transceiver design, though 24-hour lab access was provided so that students could work outside of the scheduled lab period if desired. The students had been give one previous lecture providing a very high-level overview of the concepts of frequency-division multiple access (FDMA), time-division multiple-access (TDMA), and code-division multiple access (CDMA), but the choice of which of these schemes to use was up to them.

\section{Assignment of Students into Groups}

Since multiple students had to make use of a single multiuser transmitter for development, debugging, and testing their design, it would have been quite challenging both logistically and technically for the whole class of 18 students to share one multiuser transmitter. Consequently, 
the students were split into 3 groups of 6 students, and each of the 3 groups was provided a single multiuser transmitter to share among the 6 students. Within each group of 6 students, the students were asked to form pairs, with each pair of students constituting a team. This partition is shown in Fig. 3.

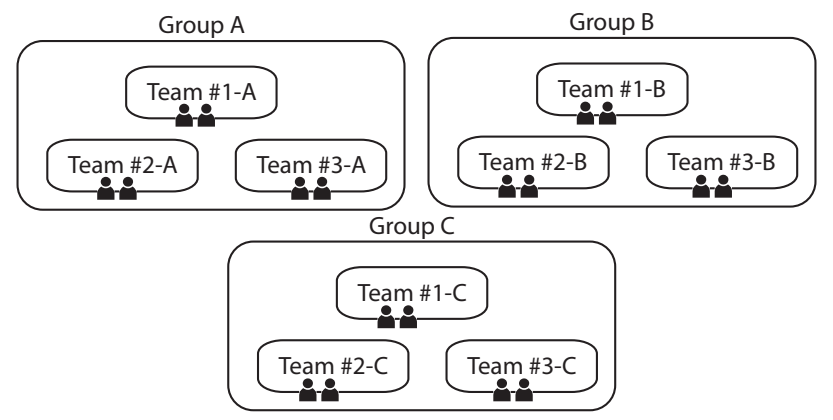

Figure 3: Partitioning of 18 students into groups and teams

In a previous offering of $\mathrm{EE} \mathrm{460,} \mathrm{the} \mathrm{lab} \mathrm{activity} \mathrm{had} \mathrm{been} \mathrm{completed} \mathrm{in} \mathrm{a} \mathrm{class} \mathrm{with} 10$ total students. With a such a small number of students, it was not necessary to split the class into groups. In that offering, students were again paired up so there were 5 total teams, and 1 multiuser transmitter for the whole class. Generally, we feel that sharing a transmitter between 3 to 5 teams seems to work best. Systems with more than 5 users (or more than 5 teams per group) lead to excessively complicated logistics while providing little benefit from a learning perspective. While from a technical perspective the task of sharing a single multiuser transmitter is more challenging as the number of users increases, the challenge in building a system with just three users (or teams) is sufficient to illustrate the basic principles of multiuser communication.

\section{Task 1: Collaborative Exercise}

The description of the lab tasks purposefully lacked procedural details, and instead succinctly gave each group of 6 students a design goal of building a multiuser MATLAB transmitter and a corresponding receiver to send and reliably decode at least 100 bits of binary information in 10 seconds or less. A logarithmically increasing amount of extra credit was awarded for groups exceeding the prescribed minimum requirement of 100 bits in 10 seconds, up to a maximum of $10 \%$ extra credit. The students were told that they would need to demonstrate their transmitter/receiver pair on arbitrary bit sequences provided by the instructor. Students were encouraged to regularly show their results to the instructor, which provided another opportunity for feedback and re-directing the students if necessary. They were not instructed to use any particular multiple-access scheme, and were free to choose their own approach.

\section{Task 2: Competitive Exercise}

In this portion of the laboratory exercise, teams within a group competed with one another, with the goal of trying to achieve the highest individual data rate among all three teams, subject to a peak power constraint. Student teams were encouraged to create robust receivers through software improvements, and were told that they may interfere with their classmates' transmissions by sending a malicious signal. Full credit was given to any team that presented a compelling argument for their chosen strategy (which may have included offensive elements such 
as jamming, or defensive elements such as a strong focus on robustness in low-SNR regimes). Extra credit (10\%) was awarded to the single team which successfully decoded the longest string of error-free bits.

\section{Final presentation and demonstration}

Each group of 6 students was given 15-20 minutes to present and describe both their collaborative and competitive transceiver designs, and demonstrate operation to the rest of the class. During this phase of the lab, a determination was made as to how much extra credit (if any) was awarded for the collaborative portion, as well as which teams were awarded extra credit for the competitive portion. During the demonstrations, each team was asked to show plots of the time and frequency content of their transmitted signals, and to interpret them. During testing, the transmitter ran on a single PC operated by the instructor, while each of the receivers ran on PCs operated by each student pair.

After the initial testing of all students' transceivers, a "grand challenge" was held, where the winning teams from each group in the competitive portion competed against each other, alongside a transceiver created by the instructor. While no points were awarded for this challenge, it did generate excitement among the students since it pitted the students against the instructor.

Following the student presentations and determination of points, the instructor gave a lecture which related concepts that the students "discovered" on their own to actual communication system principles. For example, in the competitive portion of the lab, many of the students unknowingly designed spread-spectrum systems that are known to be resilient to noise, and a brief discussion of spread-spectrum techniques was provided. The instructor also described the approach he used in the design of his competitive transceiver, which served as a retrospective summary of the project, while also a preview of topics to come in lecture.

\section{Qualitative Evaluation}

The qualitative evaluation was based on verbal interactions with students, comments on anonymous end-of-course surveys, and contents of student lab reports.

\section{Task 1: Collaborative Exercise}

In the collaborative portion of the exercise, groups A and B employed CDMA, group C employed FDMA, and no groups employed TDMA. While the groups that employed CDMA designed their transceivers from scratch, the group employing FDMA made heavy use of existing single-user transmitters that they had designed in a previous course for a similar acoustic testbed. In fact, each of the three teams in the FDMA group appeared to work in a rather distributed manner; they initially divided up the frequency spectrum, and assigned a different band of frequencies to each of the three teams within the group. As such, the specific transmitter and receiver implementation were quite different from team-to-team within the FDMA group, suggesting that each team within this group contributed an equal amount of work. The CDMA groups, on the other hand, had much more uniform transceivers from team-to-team; because CDMA requires higher levels of coordination and timing, the uniformity required in transmitters and receivers did not appear to permit the same level of distributed development demonstrated by the group which chose FDMA. 
For example, it is possible that the CDMA group transceiver designs may have been the result of an intense effort by a small subset of the group, with other students left by the wayside to help with more menial tasks like experimental setup, microphone adjustment, etc.

\section{Task 2: Competitive Exercise}

In evaluating the student lab reports, it was clear that this lab module led students to immediately be confronted with - and find their own solutions to - the issue of mitigating interference and noise in digital communication systems, while also possibly contributing controlled interference. Every student team had a different transceiver design, though there were similarities. A number of students employed spread-spectrum approaches, hoping that their classmates would use narrowband communications. Many students conducted research, primarily using online sources, to identify and borrow existing schemes which were known to be robust.

\section{Final presentation and demonstration}

Student feedback indicated appreciation for the chance to learn about one another's transceiver designs during the presentations. High levels of active student engagement were observed during the question-and-answer session. A large number of students also indicated that the competitive nature and chance for extra credit provided a lot of motivation, resulting in significant extra time being spent outside the designated lab period. Most student groups far exceeded the required design challenge of transmitting 100 bits in 10 seconds, with the winning group collaboratively transmitting at a sum rate of 3300 total bits in 10 seconds (1100 bits per user). Numerous students expressed appreciation for the lecture following the presentations that validated some of their chosen design decisions, and were particularly interested in jamming techniques.

\section{Quantitative Evaluation}

Prior to the end of the course, an anonymous survey was administered to students to gauge their opinions about the lab. Although 18 students were enrolled in the course, only 17 completed the survey.

\section{Survey results regarding collaboration}

As indicated in Fig. 4, the effect of collaboration on students' self-reported learning was mixed but promising. $71 \%$ of the class responded that collaboration in the lab was a positive learning experience, with $24 \%$ neutral. Comparing this to the impact of collaboration on students' motivation and ability to share workloads reveals a correlation which may explain why nearly a third of the class did not feel positively about their learning experience.

According to the survey, while $65 \%$ did not feel less motivated by the inclusion of collaboration, $12 \%$ were neutral and $24 \%$ were demotivated. Furthermore, just $18 \%$ of the class felt that collaboration made everyone learn and participate equally, and $82 \%$ felt that the bulk of the work was done by only a few students in their 6-person group. The question of whether some students felt demotivated because they saw others in their group taking on most of the work, or whether their lack of motivation caused others to pick up their slack initially is not clear from the survey and would require additional research to uncover such a relationship if one exists. 
Degree to which the collaborative portion of the lab...

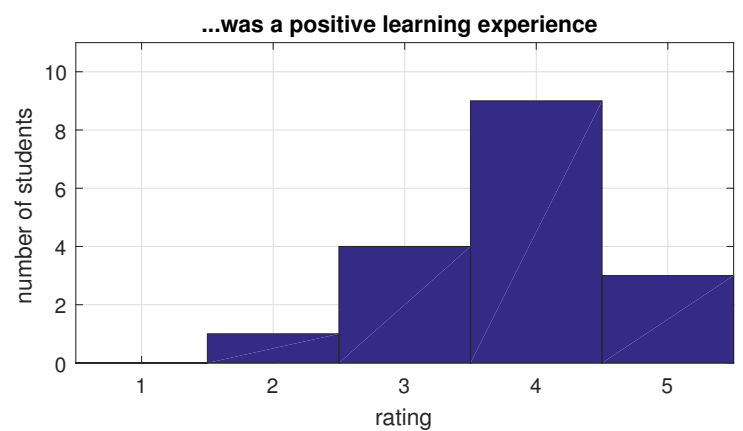

...resulted in all students in a 6-person group learning and participating

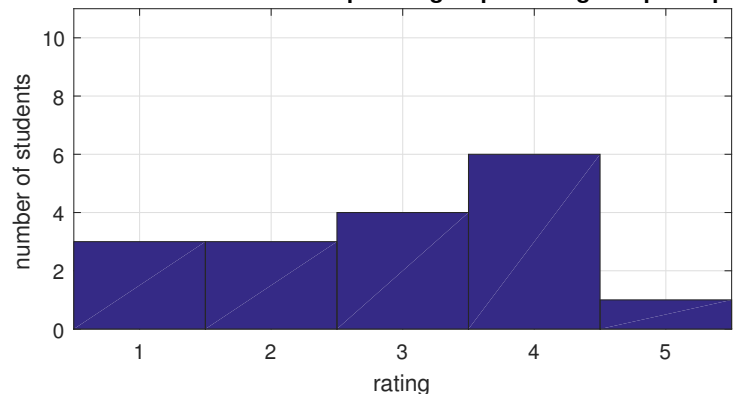

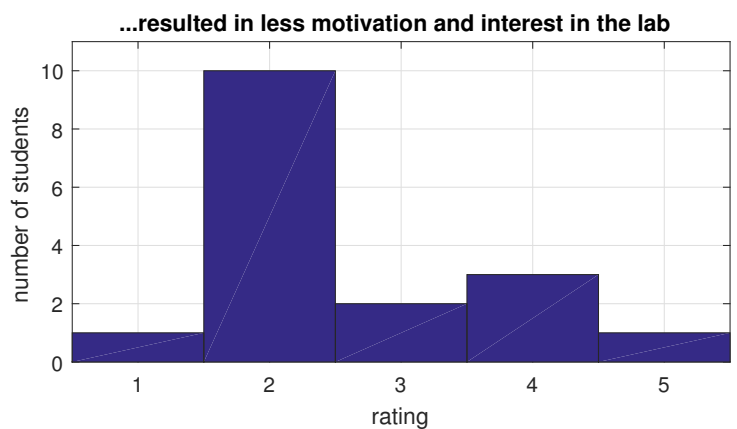

...led to a few students in each 6 person group doing most of the work

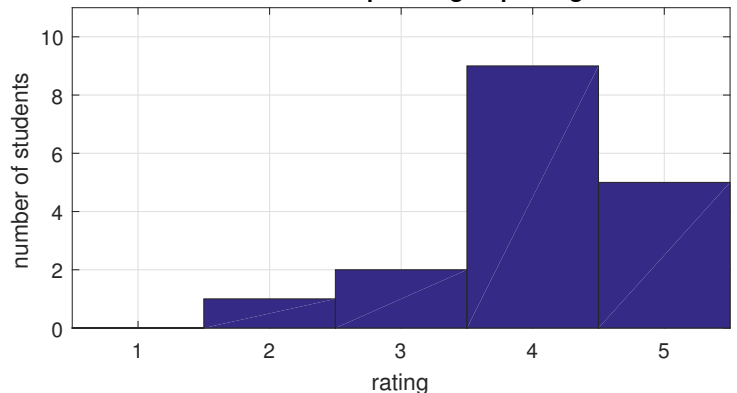

Figure 4: Survey results from fall 2016 offering of EE 460. Responses range from "1: Strongly disagree" to "5: Strongly agree" with $N=17$.

Degree to which the competitive portion of the lab...
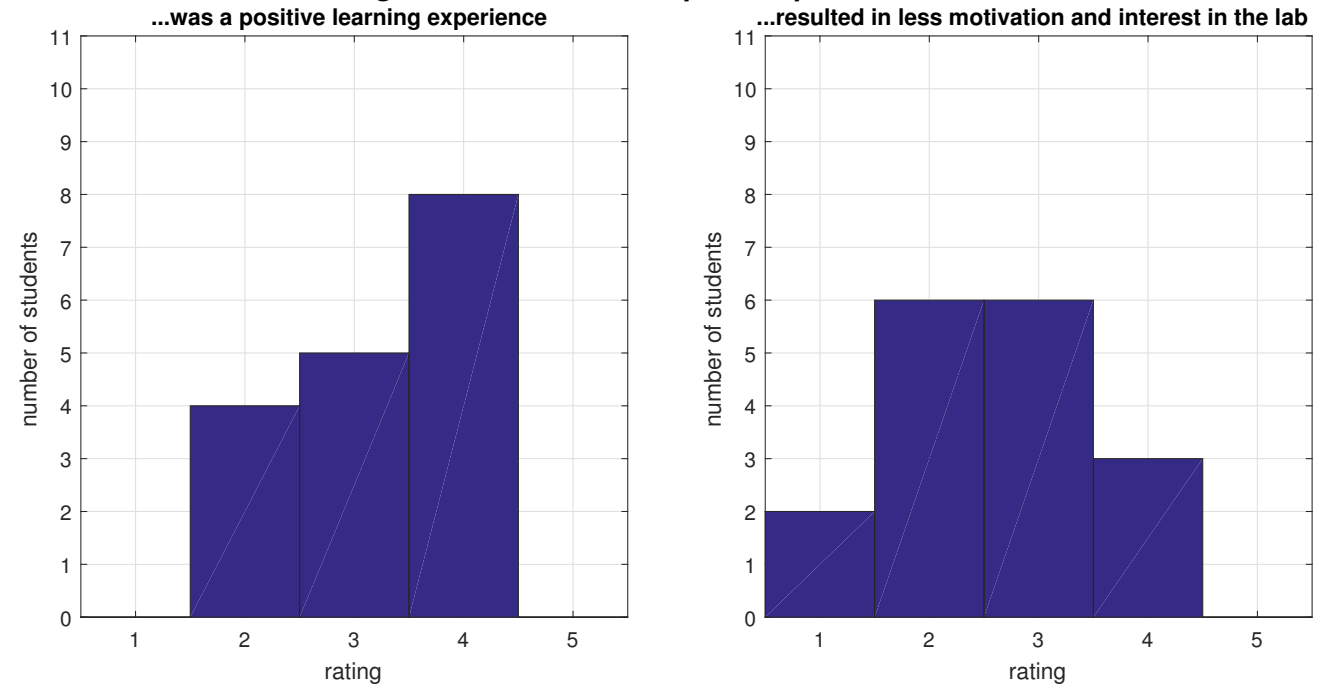

Figure 5: Survey results from fall 2016 offering of EE 460. Responses range from "1: Strongly disagree" to "5: Strongly agree" with $N=17$. 


\section{Survey results regarding competition}

Generally speaking, most students reacted less positively toward the competitive portion of the lab than the collaborative portion. Only $47 \%$ of students felt that it was a positive learning experience with $29 \%$ neutral as shown in Fig. 5, compared to $71 \%$ responding positively and $24 \%$ neutrally for the collaborative component. $47 \%$ said that they did not feel less motivated by competition, $35 \%$ were neutral, and $17 \%$ felt demotivated (compared to $65 \%, 12 \%$, and $24 \%$ for collaboration, respectively.) This is somewhat unexpected, as it indicates that competition had less of an effect on motivation than collaboration and also that collaboration was more demotivating. The result is surprising because previous literature on competition in education ${ }^{3,6,7,8}$ has suggested that competition may be polarizing to students' motivation, pushing a select group of ambitious students to succeed and discouraging others who have fallen behind.

Interestingly, no students felt that they learned more from the competitive part of the lab, with $82 \%$ responding that they learned more from the collaborative portion and $18 \%$ responding that the learned equally well from both. These results are shown in Fig. 6.

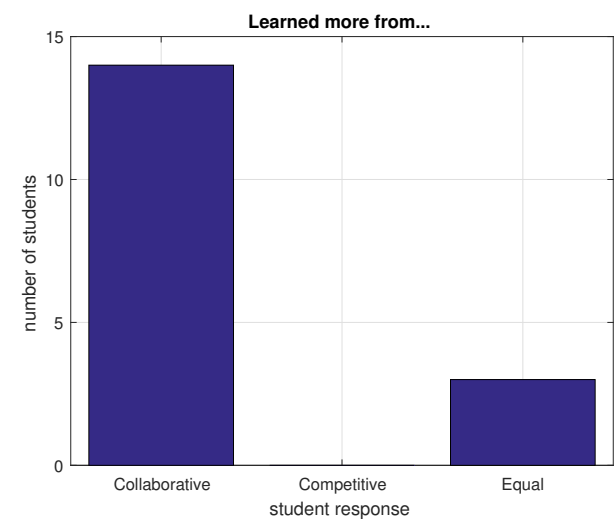

Figure 6: Survey results from fall 2016 offering of EE 460.

\section{Survey results regarding the lab overall}

Based on the survey, a large majority of students felt that the lab as a whole helped them be more successful in the course. As shown in Fig. 7, 88\% of students felt that the lab significantly improved their understanding of multiuser communications concepts. $82 \%$ agreed that the lab helped connect theory to application, and $76 \%$ said that it helped provide context for the lectures. The exact significance of these responses are unclear, as any well-designed lab would likely accomplish the same goals to some extent whether or not collaboration and competition were involved. However, these results do reinforce the widely accepted belief that both collaboration and competition in laboratory exercises foster learning.

When asked about how the lab exercise impacted class dynamics in general, $53 \%$ responded 'no change', 35\% responded 'positively', and 12\% responded 'negatively'.

The results presented by the survey suggest that while collaboration was the dominant positive contributor to students' perception of their learning, it also introduced the unforeseen issue of allowing certain students to perform more work than others without consequence. Additionally, 

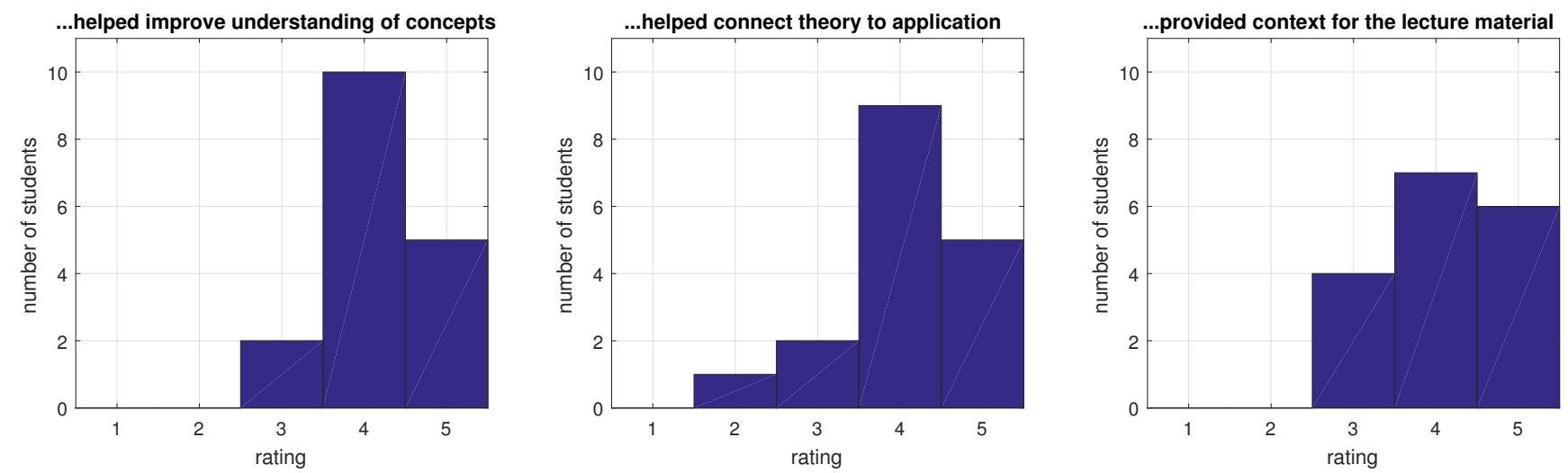

Figure 7: Survey results from fall 2016 offering of EE 460. Responses range from "1: Strongly disagree" to "5: Strongly agree" with $N=17$.

although students in the course did not feel that competition fostered learning as well as collaboration, fewer students felt demotivated by competition than collaboration. These are findings which deviate from the existing literature somewhat, because some studies suggest that competition may have a negative effect on motivation ${ }^{3,6,7,8}$ while very few discuss potential pitfalls of encouraging student collaboration.

It is also notable that despite the apparent workload inequality among students who were supposed to be collaborating, this was not reflected in perceptions of change in the class dynamic. This could suggest either that most students willingly participated in the workload inequality and were happy with the outcome (although an additional study would be required to find out why and to what extent the inequality occurred) or that a precedent had already been set for this in other courses, which would again require more investigation.

\section{Conclusions and future directions}

In this paper, we presented an inquiry based laboratory exercise for introducing multiuser communications concepts, and we studied the effects of collaboration and competition within this exercise. Although we found that most students felt that they had been positively impacted by both collaboration and competition, the majority appeared to prefer collaboration.

Intriguingly, despite students' positive responses toward the learning experience of collaboration, a majority also reported feeling that the collaborative portion of the lab caused a few students to do most of the work in each group. Similarly, a minority of students responded that collaboration caused each group member to learn and participate equally. Additionally, more students felt demotivated during the collaborative portion than the competitive portion, which is a result contrary to most existing literature but could explain the disparity in effort with further research. 
This apparent discrepancy between survey questions is notable, and it is worth investigating why most students would feel that collaboration had benefited their learning even while admitting that the bulk of the work had been done by only a few people. Although the answer is beyond the scope of this paper, some possibilities may be that some students enjoyed letting others be in control of the work, or that the students who felt left out of participating were able to learn indirectly from others' collaboration in ways not uncovered by the survey questions. In addition, repeating the study with a large class size may provide more information about the relationships between classmates and groups. Although the student-centered nature of the exercise places a relatively large demand on the instructor to answer questions and give feedback, it could potentially be scalable to larger class sizes (possibly introducing more interesting collaboration/competition effects) given additional resources.

Because of our relatively small sample size $(N=17)$ combined with our reliance on student self-reporting (which is known to be problematic), more follow-up studies are needed to draw definitive conclusions about how collaboration and competition affects student learning. It would be beneficial to introduce a baseline study as well as separate collaboration and competition between trials to better differentiate their effects. Moreover, we could add peer evaluation as part of the exercise to minimize the undesirable situation where a small number of students does a large percentage of the work.

Nevertheless, the results in this work-in-progress paper suggest that students found value in both collaboration and competition when presented in an inquiry-based lab format. By changing the group dynamics in this way, we were able to encourage a high level of student engagement and facilitate a learning experience that served to connect lecture material with real-world applications.

\section{References}

[1] M. Prince, "Does active learning work? A review of the research," Journal of Engineering Education, vol. 93, no. 3, pp. 223-231, 2004.

[2] S. Freeman, S. L. Eddy, M. McDonough, M. K. Smith, N. Okoroafor, H. Jordt, and M. P. Wenderoth, "Active learning increases student performance in science, engineering, and mathematics," Proceedings of the National Academy of Sciences, vol. 111, no. 23, pp. 8410-8415, 2014. [Online]. Available: http://www.pnas.org/content/111/23/8410.abstract

[3] C. A. Bodnar, D. Anastasio, J. A. Enszer, and D. D. Burkey, "Engineers at play: games as teaching tools for undergraduate engineering students," Journal of Engineering Education, 2016.

[4] J. Michael, "Where's the evidence that active learning works?" Advances in Physiology Education, vol. 30, no. 4, pp. 159-167, 2006. [Online]. Available: http://advan.physiology.org/content/30/4/159

[5] K. Bruffee, Collaborative Learning: Higher Education, Interdependence, and the Authority of Knowledge. Johns Hopkins University Press, 1999. [Online]. Available: https://books.google.com/books?id=n8MJVZHdULAC 
[6] M. D. Hanus and J. Fox, "Assessing the effects of gamification in the classroom: A longitudinal study on intrinsic motivation, social comparison, satisfaction, effort, and academic performance," Computers \& Education, vol. 80, pp. 152 - 161, 2015. [Online]. Available:

//www.sciencedirect.com/science/article/pii/S0360131514002000

[7] N. Mokadam, R. Lee, A. Vaporciyan, J. Walker, R. Cerfolio, J. Hermsen, C. Baker, R. Mark, L. Aloia, D. Enter, A. Carpenter, M. Moon, E. Verrier, and J. Fann, "Gamification in thoracic surgical education: Using competition to fuel performance," Journal of Thoracic and Cardiovascular Surgery, vol. 150, no. 5, pp. 1052-1058, 112015.

[8] R. Brouwer, "When competition is the loser: The indirect effect of intra-team competition on team performance through task complexity, team conflict and psychological safety," in 2016 49th Hawaii International Conference on System Sciences (HICSS), Jan 2016, pp. 1348-1357.

[9] Z. Qin, D. W. Johnson, and R. T. Johnson, "Cooperative versus competitive efforts and problem solving," Review of Educational Research, vol. 65, no. 2, pp. 129-143, 1995. [Online]. Available: http://dx.doi.org/10.3102/00346543065002129

[10] M. G. Barchilon, "Attracting engineering faculty to new frontiers in education using competition and collaboration in the classroom," in Proc. Frontiers in Education Conference (FIE'96), vol. 3, Nov. 1996, pp. $1425-1428$.

[11] G. Valença, C. Alves, V. Heimann, S. Jansen, and S. Brinkkemper, "Competition and collaboration in requirements engineering: A case study of an emerging software ecosystem," in 2014 IEEE 22nd International Requirements Engineering Conference (RE), Aug 2014, pp. 384-393.

[12] N. D. Poor, "Collaboration via cooperation and competition: Small community clustering in an MMO," in 2014 47th Hawaii International Conference on System Sciences, Jan 2014, pp. 1695-1704.

[13] A. G. Klein, "An inquiry-based acoustic signal processing lab module for introducing digital communications," in 2015 IEEE Signal Processing and Signal Processing Education Workshop (SP/SPE), Aug 2015, pp. 71-76. 